\title{
Granulocyte to Lymphocyte Ratio among Different Categories of Neonatal Sepsis according to their Vitamin D Status
}

\author{
Mirza Sultan Ahmad, Hadia Farooq, Rida Fatima and Sumaira Noor Maham \\ Department of Pediatrics, Fazle Omar Hospital, Rabwah, Pakistan
}

\begin{abstract}
The objective of this study was to ascertain whether, among the cases of neonatal sepsis, there is any significant difference between GLR (Granulocyte to Lymphocyte ratio) of different groups of patients according to their vitamin $D$ status. One hundred and nine neonates with odd admission number, admitted in NICU (Neonatal Intensive Care Unit) with clinical manifestations of neonatal sepsis during the study period from December 2017 to December 2018, were included in the study. Vitamin D deficiency was present in $83(76.1 \%)$, normal vitamin D levels were present in 21 (19.3\%), and hypervitaminosis D was detected in $5(4.6 \%)$ patients. Kruskal-Wallis test showed that there was no significant difference between GLR of different group of patients, according to their vitamin D status. There was no significant difference between GLR of different groups, according to outcome.
\end{abstract}

Key Words: Granulocyte to Lymphocyte Ratio, Vitamin D, Neonatal sepsis.

How to cite this article: Ahmad MS, Farooq H, Fatima R, Maham SN. Granulocyte to Lymphocyte Ratio among Different Categories of Neonatal Sepsis according to their Vitamin D Status. J Coll Physicians Surg Pak 2021; 31(07):871-872.

Vitamin D performs multiple functions, besides controlling calcium metabolism. It improves innate immunity and controls infections by different pathogens. This vitamin reduces the levels of pro-inflammatory cytokines, e.g. IL2 (interleukin 2), IL-6, IL-8 and tumor necrosis factor alpha. It increases levels of IL-10, which has got anti-inflammatory properties. Vitamin $\mathrm{D}$ is one of the important factors, which can modulate inflammatory response of the body. ${ }^{1}$

Many tests are being used to ascertain the inflammatory response. GLR (granulocyte tolymphocyteratio) isaninexpensive and useful test to gauge inflammation and for the diagnosis of neonatal sepsis. Alkan Ozdemir et al. used the cutoff point of 1.77 for the cases of culture proven neonatal sepsis. The sensitivity and specificity were 0.73 and 0.78 , respectively. ${ }^{2}$

Keeping in view the above mentioned studies, there was a need to ascertain whether there is a significant different between inflammatory response in different groups of the patients of neonatal sepsis according to their vitamin D status.

Correspondence to: Dr. Mirza Sultan Ahmad, Department of Pediatrics, Fazle Omar Hospital, Rabwah, Pakistan

E-mail: ahmadmirzasultan@gmail.com

Received: August 13, 2020; Revised: January 29, 2021;

Accepted: February 25, 2021

DOI: https://doi.org/10.29271/jcpsp.2021.07.871
The objective of this study was to ascertain whether, among the cases of neonatal sepsis, there is any significant difference between GLR of different groups of patients according to their vitamin $D$ status, i.e. in patients with vitamin $D$ deficiency, normal vitamin D levels, hypervitaminosis D; and to ascertain whether GLR of the patients who suffered mortality is any significantly different from the patients whosurvived.

This was prospective analytic study, conducted at Fazle Omar Hospital, Rabwah, Pakistan from December 2017 to December 2018. Inclusion criteria was neonates with odd admission number admitted in NICU (Neonatal intensive care unit) with clinical manifestations of neonatal sepsis during the study period. Ethical Committee of Fazle Omar Hospital approved the study.

The laboratory tests done on admission included FBC (full blood count), CRP (C-reactive protein), serum electrolytes, i.e. urea, creatinine, potassium, sodium, chloride, and calcium, blood culture and sensitivity, urine routine examination, urine culture, CSF examination and culture in those patients who showed neurological manifestations, and vitamin D levels.

Data analysis was done by Statistical Package for Social Sciences (SPSS) version 20. Name of the patient, age, gender (male or female), diagnosis, outcome (discharged, expired or left against medical advice [LAMA]), vitamin D levels, status of vitamin $\mathrm{D}$. Patients were divided in three categories according to their vitamin D levels. If level of vitamin D was below $20 \mathrm{ng} / \mathrm{ml}$, it was taken as vitamin D deficiency, levels between 20 and 50 $\mathrm{ng} / \mathrm{ml}$ was labelled normal, and levels above $50 \mathrm{ng} / \mathrm{ml}$ was cate- 
gorised as hypervitaminosis D. Serum and FBC reports, on admission, were entered on a data sheet. GLR ratio was shown to have non-normal distribution, therefore, median values were used for central tendency and interquartile ranges were used to show spread of the variable. Categorical variables were expressed as percentages. Kruskal-Wallis test was used to compare continuous variables with non-normal distribution of more than two groups. P-value $<0.05$ was taken as significant.

One hundred and nine cases were included in the study. Out of these, 40 (36.7\%) were females and 69 (63.3\%) were males. Ninety-one (83.5\%) were discharged, 10 (9.2\%) left against medical advice, and 8 (7.3\%) expired. Vitamin D deficiency was present in 83 (76.1\%), normal vitamin D levels were present in $21(19.3 \%)$, and hypervitaminosis D was detected in $5(4.6 \%)$ patients. Twenty-four (22\%) of the cases included in the study had culture proven sepsis. Median granulocute to lymphocyte ratio of all the cases included in this study was 1.97 (0.90-3.13). Range was 0.18-15.50. Median GLR of the culture proven sepsis was found to be 1.26(0.71-3.11).

Table I: Mean ranks of GLR in different groups, according to vitamin D levels.

\begin{tabular}{|l|c|c|c|}
\hline+++ & Median (IQR) & $\begin{array}{c}\text { Mean ranks of } \\
\text { GLR }\end{array}$ & p-value \\
\hline Vitamin D deficiency & $2.27(1.03-3.16)$ & 58.23 & \multirow{2}{*}{0.123} \\
\cline { 1 - 3 } Normal Vitamin D & $1.12(0.65-2.19)$ & 42.43 & \\
\cline { 1 - 3 } Hypervitaminosis D & $1.43(0.64-4.54)$ & 54.20 & \\
\hline
\end{tabular}

Table I shows the median (IQR), and the mean ranks of GLR of different groups of patients according to their vitamin status, i.e. patients with vitamin D deficiency, with normal vitamin D levels and those with hyper vitaminosis $D$. There was no significant difference between GLR of different categories of patients according to their vitamin $D$ levels.

Median GLR of the patients who were discharged, LAMA, and expired were found to be $2.12(0.89-3.14), 1.39$ (0.81-2.98), and $1.31(0.86-2.70)$, respectively. Kruskal-Wallis test showed that the differencebetween GLR of these threegroups was non-significant $(p=0.652)$.

Many studies have evaluated relationship between vitamin D levels and different markers of inflammation, among normal persons and the patients suffering from different infectious and non-infectious diseases. Astudy by Chandler et al. demonstrated that, among healthy people, there was inverse correlation between vitamin $\mathrm{D}$ and inflammatory markers. When inflammatory markers were rechecked after giving vitamin D course, no significant change occurred in the levels of the inflammatory markers. ${ }^{3}$

GLR ratio is a cost-effective inflammatory marker. Keeping in view the fact that vitamin $D$ deficiency enhances inflammatory response of the body and GLR is an indicator of intensity of inflam- matory response, many studies have been conducted to check association between vitamin D level and GLR. Ekbas et al. showed negative correlation between vitamin D levels and GLR. ${ }^{4}$ Sepsis is an inflammatory response of the body to different pathogens. Neonatal sepsis is an important cause of global mortality and morbidity. This high mortality rate makesitimperative to study different factors, which modify this inflammatory response in these patients. Various studies assessed the value of GLR to assess inflammatory response of the body among these cases. This ratio was found to be positively associated with neonatal sepsis. ${ }^{5} \mathrm{~A}$ study by Can et al. showed that there is positive association between GLR and early onset neonatal sepsis. ${ }^{6}$

This study showed that there was no significant difference between GLR of different groups of cases of neonatal sepsis, according to their vitamin $D$ status.

\section{CONFLICTOF INTEREST:}

The authors declared no conflict of interest.

\section{AUTHORS' CONTRIBUTION:}

MSA: Supervised and wrote the article.

$\mathrm{HF}, \mathrm{RF}$ : Data collection.

SNM: Literature review.

\section{REFERENCES}

1. Abdel-Hady H, Yahia S, Megahed A, Mosbah A, Seif B, Nageh $E$, et al. Mediators in preterm infants with lateonset sepsis: A randomised controlled trial. J Pediatr Gastroenterol Nutr 2019; 68(4):578-84. doi: 10.1097/ MPG.0000000000002238.

2. Alkan Ozdemir S, Arun Ozer E, Ilhan O, Sutcuoglu S. Can neutrophil to lymphocyte ratio predict late-onset sepsis in preterm infants? J Clin Lab Anal 2018; 32(4):e22338. doi: 10.1002/jcla.22338.

3. Chandler PD, Scott JB, Drake BF, Kimmie Ng, Manson JE, Rifai $N$, et al. Impact of vitamin D supplementation on inflammatory markers in African Americans: Results of a four-arm, randomised, placebo-controlled trial. Cancer Prev Res (Phila) 2014; 7(2):218-25. doi: 10.1158/19406207.CAPR-13-0338-T.

4. Akbas EM, Gungor A, Ozcicek A, Akbas N, Askin S, Polat M. Vitamin $D$ and inflammation: Eevaluation with neutrophil-to-lymphocyte ratio and platelet-tolymphocyte ratio. Arch Med Sci 2016; 12(4):721-7. doi: 10.5114/aoms.2015.50625.

5. Wilar R. Diagnostic value of eosinopenia and neutrophil to lymphocyte ratio on early onset neonatal sepsis. Korean J Pediatr 2019; 62(6):217-223. doi: 10.3345/kjp. 2018. 06723.

6. Can E, Hamilcikan Ş, Can C. The value of neutrophil to lymphocyte ratio and platelet to lymphocyte ratio for detecting early-onset neonatal sepsis. J Pediatr Hematol Oncol 2018; 40(4):e229-e32. doi: 10.1097/MPH.0000000 000001059 . 